\title{
Síntomas de depresión, ansiedad y estrés post-traumático en adolescentes siete meses después del terremoto del 27 de febrero de 2010 en Chile
}

\section{Depression, anxiety and post-traumatic stress disorder symptoms in adolescents seven months after the february 272010 earthquake in Chile}

\author{
Camila A. Díaz \\ Gonzalo R. Quintana \\ $\&$ \\ Edgar H. Vogel \\ Facultad de Psicología, Universidad de Talca, Chile
}

(Rec: 23 de mayo de 2011 / Acep: 20 de noviembre de 2011)

\begin{abstract}
Resumen
El 27 de febrero de 2010, Chile vivió uno de los terremotos más fuertes registrados en la historia, provocando serias consecuencias en la salud mental de las personas. El objetivo del presente estudio fue dar una primera evaluación del impacto de dicho fenómeno en la salud mental de los adolescentes. Un total de 304 adolescentes fueron evaluados en depresión, ansiedad y trastorno de estrés post-traumático. Los resultados indican un aumento en la incidencia de todos los desórdenes, corroborando al género femenino como una población de riesgo al alcanzar puntajes significativamente mayores que los hombres en todas las escalas. Además, se demostró una estrecha relación de co-morbilidad entre los síntomas de los trastornos evaluados.

Palabras clave: Depresión, ansiedad, TEPT, adolescentes, terremoto.
\end{abstract}

\begin{abstract}
On February 27th of 2010, Chile experienced one of the strongest earthquakes in recorded history, causing serious consequences on people's mental health. The goal of the present study was to provide a preliminary assessment of the impact of this phenomenon in adolescent's mental health. A total of 304 adolescents were assessed on depression, anxiety and post-traumatic stress disorder. The results indicate an increase in the incidence of all the assessed disorders, confirming that females are a risk population who reached significantly higher scores than males on all scales. The study further demonstrated a close relationship of co-morbidity among the symptoms of the assessed disorders.
\end{abstract}

Keywords: Depression, anxiety, PTSD, adolescent, earthquake.

Correspondencia: Gonzalo R. Quintana Zunino, Facultad de Psicología, Universidad de Talca, Casilla № 747 Talca/Chile, Tel: (5671) 201519, Fax: (5671) 201510. E-mail: gonzalo.qz@gmail.com.

Los autores agradecen a Felipe Cornejo por sus enriquecedores aportes a la elaboración de esta investigación. El presente estudio fue financiado por la Universidad de Talca a través del programa de investigación “Calidad de Vida y Ambientes Saludables" (Res. 387/2007). 


\section{Introducción}

El pasado 27 de febrero de $2010(27 / F)$, un terremoto de 8.8 grados Richter azotó la zona centro sur de Chile, causando una serie de pérdidas fatales, materiales, puestos de trabajo y destrucción del ambiente inmediato (e.g. Bresciani, 2010). Al igual que en otros desastres naturales, en este evento probablemente también se han desencadenado consecuencias directas e indirectas en la salud física y psicológica tanto a corto como a mediano y largo plazo, desafiando los recursos físicos y cognitivos de cualquier persona. Diversos estudios han demostrado que el impacto en la salud mental no es igual para todos, siendo consideradas como poblaciones de riesgo las mujeres (Tolin \& Foa, 2006), los niños y adolescentes (Groome \& Soureti, 2004), los ancianos (Ticehurst, Webster, Carr \& Lewin, 1996) y las personas de escasos recursos (Kar, Mohapatra, Nayak, Pattanaik, Swain \& Kar, 2007).

Dentro de los trastornos registrados por la literatura en este tipo de fenómenos, los más estudiados son la depresión, la ansiedad y el trastorno de estrés postraumático (TEPT), los cuales entre sí tienen una alta superposición y co-ocurrencia de los síntomas (Stefanis \& Stefanis, 2002). No obstante, en este tipo de eventos el TEPT sería el más prevalente y aquel relacionado a una mayor co-morbilidad (Norris \& Elrod, 2006 citado en Norris, Galea, Friedman \& Watson, 2006; Norris, Friedman \& Watson, 2002).

La Tabla 1 presenta datos de prevalencia de cada trastorno para ambos géneros, según lo estipulado por el manual estadístico y diagnóstico de los trastornos mentales IV (DSM-IV-TR; 2000), junto al de estudios realizados en muestras representativas de Chile (Araya, Rojas, Fritsch, Acuña \& Lewis, 2001; Pérez et al., 2009; Vicente, Rioseco, Saldivia \& Torres, 2002). Las cifras señaladas en la tabla se verían seriamente afectadas después de un evento traumático, aumentando de forma considerable cada uno de ellos (e,g. Cairo, Dutta \& Nawaz, 2010; Thienkrua et al., 2007), pero variando de fenómeno en fenómeno por sus características particulares.

La situación no es muy diferente en niños y adolescentes, donde la alta prevalencia de depresión (Goenjian, 1995), ansiedad (Leen-Feldner, Feldner, Reardon, Babson \& Dixon, 2008) y TEPT (Eksi et al., 2007) frente a la exposición de un evento traumático es una constante, junto a las diferencias por género (Bokszczanin, 2007), tanto en desastres naturales como en eventos traumáticos provocados por el hombre, tales como bombardeos masivos (Norris, Friedman, Watson, Byrne, Díaz \& Kaniasty, 2002).

Posteriormente a un evento traumático, el inicio de los síntomas psicológicos, curso y duración es variable, pudiendo incluso retardarse su inicio varios meses desde el momento del trauma (e.g. Priebe, Grappasonni, Mari, Dewey, Petrelli \& Costa, 2009). Teniendo en cuenta que en una primera instancia los síntomas relacionados con este tipo de eventos deben comprenderse como una respuesta adaptativa exacerbada del organismo frente a las contingencias del entorno (Cohen, 2008), es principalmente el tiempo que estas se mantienen lo que las hace poco adaptativas y nocivas para la persona. Según el DSM-IV-TR (2000), el diagnóstico de la depresión está dado por el catastro de una serie de síntomas de anhedonia, autoestima negativa, entre otros, sostenidos la mayor parte del día por al menos dos semanas; trastorno que normalmente dura alrededor de 6 meses desde su inicio. En el caso de la ansiedad, su diagnóstico se caracteriza por la presencia de síntomas de preocupación, falta de concentración y manifestaciones fisiológicas (falta de sueño, sudoración excesiva, etc.) superior a 6 meses, pudiendo llegar a ser crónico para algunas personas. Respecto al TEPT, su diagnóstico se caracteriza por la presencia de síntomas de re-experimentación, evitación del objeto gatillador y la activación fisiológica de la persona durante menos de 3 meses (TEPT agudo) o más (TEPT crónico), pudiendo incluso retrasarse 6 meses o más (TEPT de inicio demorado); pudiendo durar normalmente 3 meses e incluso más de un año.

Finalmente, considerando el costo que este tipo de enfermedades mentales puede tener para la salud pública de un país (Figueroa, González \& Torres, 2010) y las consecuencias colaterales que merman el presupuesto asignado a la salud mental (e.g. gasto en infraestructura; Shoaf \& Rottman, 2000), se hace imprescindible el catastro del inicio como del curso temporal de dichos trastornos (Neria, Besser, Kiper \& Westphal, 2010) y de aquellas características personales (Benight, Swift, Sanger, Smith \& Zeppelin, 1999), interpersonales y sociales (Bokszczanin, 2008) que puedan

Tabla 1. Prevalencia normal por trastorno y género en base al DSM-IV y a estudios en muestras chilenas representativas.

\begin{tabular}{ccccccc}
\hline & & DSM-IV & Araya et al., 2001 & Vicente et al., 2002 & Pérez et al., 2009 \\
\hline \multirow{2}{*}{ Depresión } & Mujeres & $10-25$ & 8.0 & 11.3 & - \\
\cline { 2 - 6 } & Hombres & $5-12$ & 2.7 & 6.4 & - \\
\hline \multirow{2}{*}{ Ansiedad } & Mujeres & \multirow{2}{*}{$3-5$} & 6.9 & 4.1 & - \\
\cline { 2 - 6 } & Hombres & & \multirow{2}{*}{$1-14$} & 3.2 & 0.9 & 6.2 \\
\cline { 2 - 6 } TEPT & Mujeres & & - & 6.2 & 2.5 \\
\cline { 2 - 6 } & Hombres & & - & 2.5 & - \\
\hline
\end{tabular}


prevenirlo, mitigarlo, perdurarlo o incluso agravarlo en pos de una oportuna intervención y tratamiento.

Así, a pesar que la evidencia empírica sobre las consecuencias psicológicas en distintos tipos de poblaciones a partir de la experiencia de un evento traumático es vasta y diversa (Neria et al., 2010; Norris, Friedman \& Watson, 2002; Norris, Friedman, Watson, Byrne, Díaz \& Kaniasty, 2002), a más un año del terremoto del 27 de febrero, la mayoría de la literatura sobre este fenómeno versa sobre ciencias de la ingeniería y física de la tierra. En lo que respecta a la psicología, sólo existen dos estudios que han evaluado el impacto de dicho fenómeno, uno en salud mental (Leiva $\&$ Quintana, 2010) y otros en variables relacionadas al ejercicio laboral (Jiménez \& Cubillos, 2010), ambos en adultos. Por tanto, la presente investigación pretende evaluar el impacto psicológico siete meses después del desastre a través del análisis de los trastornos más prevalentes desarrollados después de un evento traumático como lo son la depresión, ansiedad y TEPT en adolescentes expuestos al terremoto.

\section{Método}

\section{Participantes}

La muestra estuvo constituida por 304 estudiantes entre $8^{\mathrm{vo}} \mathrm{y}^{\text {to }}$ medio de 3 colegios de la ciudad de Chillán (hombres $=42.1 \%$; mujeres $=57.9 \%$ ), cuya edad media fue de 15.3 años ( $\mathrm{DS}=1.25)$ en un rango de 13 a 19 años. Del total, un $66.8 \%$ se encontraba en Chillán al momento del terremoto, mientras que los demás se encontraban en diversas localidades cercanas de la octava región (e.g. Bulnes y San Carlos), salvo casos aislados, en donde la intensidad del terremoto fue de 8.8 Richter y 8 en la escala Mercalli.

Un $37.8 \%$ de los adolescentes declararon haber tenido una pérdida de enseres o bienes materiales a raíz del terremoto, mientras que un $26.6 \%$ de ellos reportó que su casa resultó dañada por el terremoto. Por otro lado, sólo un 1.6\% reportó haber sufrido daños físicos considerables como consecuencia del terremoto (fracturas, hospitalización, etc.), aumentando a un $6.6 \%$ cuando se trataba de un familiar. Finalmente, un $2.6 \%$ de ellos sufrieron la muerte de un ser querido (familiares o personas cercanas).

\section{Instrumentos}

Para este estudio se utilizaron las siguientes escalas:

a) El Inventario de Depresión de Niños (Kovacs, 1981; Stark \& Laurent, 2001). Es una escala de auto-reporte de 27 ítems con tres alternativas de respuesta $(0=$ "no me ocurre", 1 = "me ocurre poco" y 2 = "me ocurre mucho"), destinada a medir la presencia de síntomas de depresión en niños y adolescentes, a quienes se les pide escoger una de las alternativas en cada ítem en términos de las "últimas dos semanas". El inventario posee cinco dimensiones $(\alpha=.904)$ : "Anhedonia" compuesta por ocho ítems $(\alpha=.682)$, "Estados de ánimo negativo" por cinco $(\alpha=.734)$, "Inefectividad" por cuatro $(\alpha=$ $.652)$, "Problemas interpersonales" por tres $(\alpha=.248)$ y "Autoestima negativa" por siete $(\alpha=.762)$. Según el manual del inventario (Kovacs, 1992), en base a una población estandarizada $(\mu=10.5 ; \sigma=7.3)$, el puntaje de corte para establecer quién tiene una "Alta" o "Baja" presencia de síntomas se estableció como una desviación estándar por sobre la media. Por lo tanto, el puntaje de corte utilizado en la presente investigación fue de 18.

b) La Escala Revisada de Ansiedad Manifiesta para Niños (Reynolds \& Richmond, 1978; Stark \& Laurent, 2001). Es una escala de auto-reporte de 37 ítems de respuesta dicotómica (SÍ o NO) destinada a medir la presencia de síntomas de ansiedad en niños y adolescentes, a quienes se les pide determinar si cada una de las afirmaciones lo describe o no. La escala posee cuatro dimensiones $(\alpha=.799)$ : "Ansiedad Fisiológica" compuesta por diez ítems $(\alpha=.696)$, "Preocupación/Sobresensibilidad" por once $(\alpha=.791)$, "Concentración" por siete $(\alpha=.677)$ y "Mentira" por nueve $(\alpha=.685)$. Según el manual de Reynolds y Richmond (1985, p. 20), para el cálculo del puntaje de corte sobre quién tiene una "Alta" o "Baja" presencia de síntomas, se utilizó una desviación estándar en base a una población estandarizada $(\mu=11.7 ; \sigma=$ 6.21). Por lo tanto, el puntaje de corte utilizado en la presente investigación fue de 18 puntos.

c) Escala de TEPT de Niños (Foa, Cashman Jaycox \& Perry, 1997; Foa, Johnson, Fenny \& Treadwell, 2001). Es una escala de auto-reporte que consta de 24 ítems, 17 de ellos describen cada uno de los síntomas presentes en el DSM-IV-TR (2000) para TEPT destinado a medir la presencia de sus síntomas después de la exposición a un amplio rango de eventos traumáticos en niños y adolescentes. Adicionalmente, la escala cuenta con siete ítems para evaluar el funcionamiento diario (e.g. relaciones con amigos, en las tareas del colegio) en donde el evaluado responde Sí o No en relación a si los problemas descritos en los ítems contestados anteriormente han interferido en alguna de esas actividades. La escala posee tres dimensiones $(\alpha=.914)$ : "Reexperimentación" compuesta por cinco ítems ( $\alpha=.849)$, "Evitación" por siete $(\alpha=.815)$ y "Aumento de la Activación" por cinco $(\alpha=.759)$. La escala posee cuatro alternativas de respuesta $(0=$ "No, en absoluto o sólo una vez", 1 = "Una vez por semana o menos / de vez en cuando," 2 = "2 a 4 veces a la semana / la mitad del tiempo" y $3=$ "5 o más veces a la semana / casi siempre"). Para el cálculo del puntaje, los autores sugieren calificar con "Bajo nivel de sintomas de TEPT" puntajes menores o iguales a 17 y "Alto nivel de sintomas de TEPT" aquellos puntajes mayores a 17. 
Estas escalas fueron escogidas dado que son unas de las más utilizadas en este tipo de evaluación (eventos traumáticos), cumplen con buenas propiedades psicométricas y son de auto-reporte, lo que facilita su aplicación masiva.

Teniendo en cuenta que las escalas utilizadas no tienen una versión adaptada al contexto chileno, se procedió a realizar un proceso de retro-traducción de las escalas. Un psicólogo con habilidades avanzadas en la lengua inglesa tradujo literalmente las escalas al español para conservar la naturaleza de los ítems. Posteriormente, se pidió a 3 psicólogos que evaluaran la pertinencia de la traducción ítem por ítem y la adecuación al contexto chileno. Posteriormente, recogiendo todas las sugerencias de los evaluadores, se le pidió a un traductor profesional que tradujera las escalas al inglés. Finalmente, se volvió a pedir a los evaluadores que juzgaran como "Adecuado" o "No Adecuado" si los ítems se encuentran bien traducidos, contextualizados y adecuados tanto lingüística como conceptualmente. Respecto a la escala de TEPT, si bien existe una evaluación preliminar (Bustos, Rincón \& Aedo, 2009), se utilizó la codificación de la escala original y los resultados preliminares de la validez de la escala indican que posee índices similares, así como las demás escalas.

\section{Procedimiento}

En una primera instancia se contactó a las instituciones educativas, coordinando la evaluación, ya sea con el jefe de UTP u orientador de los distintos establecimientos, quienes colaboraron con disponibilidad de horario de cada uno de los cursos, realizándose las evaluaciones entre el 23 de septiembre y el 14 de octubre de 2010.

Se diseñó un consentimiento informado para los padres, en el cual se explicitaba el objetivo de investigación, el procedimiento de evaluación y cómo serían tratados los resultados, siendo distribuido en todos los cursos. Posteriormente, y luego de dos semanas de haber entregado a los estudiantes dicho consentimiento, se procedió a la recepción de éste, en donde sólo aquellos estudiantes que devolvieron dicho documento aprobado por los padres participaron de la evaluación. El día de la aplicación se distribuyó la batería que contenía las instrucciones, tanto para los profesores como los estudiantes, junto con el registro de datos demográficos y las escalas de depresión, ansiedad y TEPT, en dicho orden, para todos los cursos. La evaluación fue supervisada por uno de los autores.

\section{Análisis de datos}

Con el fin de caracterizar la presencia de síntomas en cada uno de los trastornos evaluados, se establecieron distribuciones de frecuencia a partir de los criterios de corte definidos para cada instrumento. Posteriormente, para establecer las diferencias entre grupos se utilizó la prueba de Ji cuadrado. Finalmente, para evaluar la co-morbilidad de los síntomas de los trastornos evaluados, dado que las escalas poseen distintas codificaciones, se estandarizaron las puntuaciones en una dispersión $Z$ y se utilizó el coeficiente de correlación de Pearson.

\section{Resultados}

La Tabla 2 muestra el porcentaje de hombres y mujeres que sufrieron distintos daños y pérdidas a causa del desastre. En ella se aprecia, en una primera instancia, la baja incidencia de afectados, especialmente para el tipo de daños más severos (daños físicos, muerte de un ser querido). Adicionalmente, se puede ver que sólo en la pérdida de materiales los hombres puntúan significativamente más que las mujeres $(p<.01)$.

Por otro lado, la Tabla 3 muestra el porcentaje de adolescentes con "alta" presencia de síntomas de cada trastorno según los criterios de corte definido en la metodología. Además, se incluye como referencia una cifra de la prevalencia normal del trastorno en la población chilena. Así, teniendo en cuenta la prevalencia normal por trastorno y género, se puede observar que la presencia de síntomas de cada uno de los trastornos a partir de la exposición al terremoto del 27 de febrero aumentó, tanto a nivel total como en hombres y mujeres, salvo en el caso particular de la depresión en hombres. Junto a esto, se puede constatar que serían las mujeres quienes presentan una mayor incidencia que los hombres al haber un número significativamente mayor de mujeres que alcanzan el criterio de alta presencia

Tabla 2. Porcentaje total y por género de diversos aspectos asociados a la exposición al trauma.

\begin{tabular}{lccc}
\hline Ítem & Hombres & Mujeres & $\chi^{2}$ \\
\hline Pérdidas materiales & 20.6 & 17.6 & $9.88^{* *}$ \\
Daños en el inmueble & 13.5 & 13.5 & 2.31 \\
Daños físicos personales & 0.7 & 1 & 0.02 \\
Daños físicos familiares & 3.3 & 3.3 & 0.49 \\
Muerte de un ser querido & 1 & 1.6 & 0.08 \\
\hline
\end{tabular}

$* *=p<.01$. 
Tabla 3. Porcentaje total y por género de adolescentes con niveles altos de depresión, ansiedad y TEP.

\begin{tabular}{|c|c|c|c|c|c|}
\hline & & & & & Diferencias por Género \\
\hline & Criterio & Total & Hombres & Mujeres & $\chi^{2}$ \\
\hline \multirow{2}{*}{ Depresión } & Alta Depresión (>18 ptos.) & 13.5 & 3.1 & 21.0 & $20.345 * * *$ \\
\hline & Porcentaje de Referencia & 5 a 25 & 6.4 & 11.3 & \\
\hline \multirow{2}{*}{ Ansiedad } & Alta Ansiedad (>18 ptos.) & 29.6 & 14.1 & 40.9 & $25.628 * * *$ \\
\hline & Porcentaje de Referencia & 3 a 5 & 3.2 & 6.9 & \\
\hline \multirow{2}{*}{ TEPT } & Alto TEPT (>17 ptos.) & 20.4 & 7.8 & 29.5 & $21.559 * * *$ \\
\hline & Porcentaje de Referencia & 1 a 14 & 2.5 & 6.2 & \\
\hline
\end{tabular}

Tabla 4. Porcentaje total y por género de adolescentes que perciben disrupción en tareas cotidianas.

\begin{tabular}{|c|c|c|c|c|}
\hline Ítem & Total & Hombres & Mujeres & $\chi^{2}$ \\
\hline 18. Al rezar & 16.1 & 10.9 & 19.9 & $4.389 *$ \\
\hline 19. En las tareas y deberes en el hogar & 33.2 & 31.2 & 34.7 & .388 \\
\hline 20. En las relaciones con los amigos & 23 & 19.5 & 25.6 & 1.524 \\
\hline 21. En las cosas divertidas y hobbies & 21.7 & 19.5 & 23.3 & .618 \\
\hline 22. En las tareas & 27 & 27.3 & 26.7 & .015 \\
\hline 23. En las relaciones con tu familia & 26.6 & 17.2 & 33.5 & $10.117 * * *$ \\
\hline 24. En la felicidad general con tu vida & 32.6 & 21.9 & 40.3 & $11.507 * * *$ \\
\hline
\end{tabular}

$*=p<.05$. $* * *=p<.001$.

de síntomas en cada uno de los trastornos evaluados $(p \mathrm{~s}<$ $0.001)$.

La Tabla 4 presenta el porcentaje total y por género de adolescentes que perciben disrupción en tareas cotidianas. Allí se observa que aproximadamente un quinto de los entrevistados sufrieron algún tipo de disrupción en sus tareas cotidianas, siendo "las tareas y deberes del hogar" y "en la felicidad con tu vida", aquellos aspectos más afectados. Cabe mencionar que las mujeres manifiestan una mayor disrupción, específicamente en los ítems "al rezar", en "las relaciones con la familia" y en la "felicidad general de la vida" $(p \mathrm{~s}<.001)$.
La Tabla 5 muestra los mismos datos de la Tabla 4, pero desagregados entre aquellas personas que presentaron altos síntomas de TEP y aquellos que presentaron bajos síntomas. En la tabla se puede apreciar que todos aquellos que son diagnosticados con un "Alto TEPT" perciben significativamente una mayor disrupción en todas las tareas cotidianas que aquellos diagnosticados con un "Bajo TEPT” $(p \mathrm{~s}<.001)$.

Finalmente, por medio de un análisis de correlaciones entre los puntajes totales de escalas, se corrobora la estrecha y significativa relación de la comorbilidad de los síntomas entre los trastornos, en donde la depresión correlaciona significativamente tanto con ansiedad y TEPT $(r=.760 \mathrm{y}$

Tabla 5. Porcentaje total y por criterio de diagnóstico TEP de adolescentes que perciben disrupción en tareas cotidianas.

\begin{tabular}{lllll}
\hline Ítem & Total & Bajo TEPT & Alto TEPT & $\chi^{2}$ \\
\hline 18. Al rezar & 16.1 & 12.4 & 30.6 & $12.156^{* * *}$ \\
19. En las tareas y deberes en el hogar & 33.2 & 25.6 & 62.9 & $30.924^{* * *}$ \\
20. En las relaciones con los amigos & 23 & 16.9 & 46.8 & $24.782^{* * *}$ \\
21. En las cosas divertidas y hobbies & 21.7 & 16.5 & 41.9 & $18.744^{* * *}$ \\
22. En las tareas & 27 & 21.9 & 46.8 & $15.502^{* * *}$ \\
23. En las relaciones con tu familia & 26.6 & 21.1 & 48.4 & $18.837^{* * *}$ \\
24. En la felicidad general con tu vida & 32.6 & 21.5 & 75.8 & $66.312^{* * *}$ \\
\hline
\end{tabular}

$* * *=p<.001$. 
$r=.708$, respectivamente; $p \mathrm{~s}<.001)$ y la ansiedad correlaciona significativamente con TEPT $(r=.736 ; p<.001)$.

\section{Discusión}

Siendo el sexto terremoto más intenso registrado en la historia humana, a casi dos años, las consecuencias son evidentes. La literatura demuestra un amplio conocimiento respecto a las consecuencias en la salud mental de las personas a partir de la exposición a estos fenómenos traumáticos. Sin embargo, la mayoría de la literatura en salud mental existente sobre dicho fenómeno versa sobre vivencias (Reyes, 2010; Ortiz-Barrera \& Manzo-García, 2010), intervenciones (Loubat, Fernández \& Morales, 2010) y los posibles efectos provocados (Cova \& Rincón, 2010), sistematizados, en su mayoría, en un número especial de la revista Terapia Psicológica (Vogel \& Vera, 2010).

El presente estudio da cuenta del impacto en la salud mental de adolescentes expuestos a este terremoto. En él se corrobora el aumento en la prevalencia de síntomas de depresión, ansiedad y TEPT junto a la estrecha relación entre ellos. Además, se observaron claras diferencias por género, pues las mujeres manifiestan más síntomas y en mayor intensidad en cada uno de los trastornos evaluados. Sin embargo, en términos objetivos, se puede dar cuenta que sólo hubo diferencias significativas en la pérdida de bienes materiales entre hombres y mujeres, pero no en cuanto a daños en el hogar, daños físicos considerables propios y de parientes, y tampoco por la muerte de un familiar o persona cercana.

Estos resultados confirman hallazgos de otros eventos traumáticos y pueden contribuir a la comprensión de la severidad de los síntomas a partir del nivel de exposición al terremoto, al comparar los datos presentes con otras muestras de otras ciudades expuestas a una menor intensidad (Goenjian, Steinberg, Najarian, Fairbanks, Tashjian \& Pynoos, 2000), con efectos aditivos que pueda tener el haber experimentado también el tsunami en pobladores costeros (Leiva \& Quintana, 2010), o quizá con poblaciones en donde se haya experimentado un mayor número de réplicas y de mayor intensidad (e.g. habitantes de la ciudad de Concepción).

Por otro lado, esta investigación provee de un primer reporte sobre el impacto del terremoto en adolescentes, lo cual puede servir de antecedente para estudios posteriores que evalúen el desarrollo y duración de los trastornos aquí evaluados, donde la evidencia sugiere el declive de los mismos en un periodo relativo, el cual es moderado por distintas variables (Neria et al., 2010), teniendo en cuenta que la aparición de los síntomas pueda llegar a tomar incluso 18 meses en el caso del TEPT (Sungur \& Kaya, 2001).

Es necesario que se investiguen las consecuencias en la salud mental en otras poblaciones de riesgo poco estudiadas (ancianos, personal de salud, bomberos, rescatistas, etc.) expuestas a altos niveles de estrés que este tipo de catástrofe conlleva, y cómo esto puede llegar a afectar a los hijos de aquellas personas. Esto último es especialmente importante si se tiene en cuenta que se ha demostrado el aumento en la probabilidad de desarrollar TEPT en los niños cuyos padres sufren de dicho trastorno (Li, Huang, Tan, Liu, Zhou \& Yang, 2010) o la modulación del rol parental en el proceso de recuperación (Bokszczanin, 2008).

Adicionalmente, se hace necesario constatar el impacto que tiene el hecho que los medios de comunicación exponen y re-exponen al público a los eventos traumáticos asociados a la catástrofe, lo cual podría exacerbar la aparición o intensidad de los síntomas. Esto es relevante dado el gran porcentaje de gente que consumió información después de un evento como el terremoto del 27 de febrero y las emociones activadas por la televisión chilena en la cobertura de este fenómeno (Souza \& Martínez, 2011).

\section{Referencias}

American Psychiatric Association. (2000). Diagnostic and statistical manual of mental disorders (4th ed., text rev.). Washington, DC: Author.

Araya, R., Rojas, G., Fritsch, R., Acuña, J., \& Lewis, G. (2001). Common mental disorders in Santiago, Chile: Prevalence and socio-demographic correlatos. The British Journal of Psychiatry, 178, 228-233.

Benight, C.C., Swift, E., Sanger. J., Smith, A., \& Zeppelin, D. (1999). Coping self-efficacy as a mediator of distress following a natural disaster. Journal of Applied Social Psychology, 29, 2443-2464.

Bresciani, L. (2010). Chile 27F 2010: La catástrofe de la falta de planificación. EURE, 36, 151-153.

Bokszczanin, A. (2007). PTSD symptoms in children and adolescents 28 months after a flood: Age and gender differences. Journal of Traumatic Stress, 20, 347-351.

Bokszczanin, A. (2008). Parental support, family conflict, and overprotectiveness: Predicting PTSD symptom levels of adolescents 28 months after a natural disaster. Anxiety, Stress, \& Coping, 21, 325-335.

Bustos, P., Rincón, P., \& Aedo, J. (2009). Validación preliminar de la escala infantil de síntomas del trastorno de estrés postraumático (child PTSD symptom Scale, CPSS) en niños/as y adolescentes víctimas de violencia sexual. Psykhe, 18, 113-126.

Cairo, J.B., Dutta, S., \& Nawaz, H. (2010). The prevalence of posttraumatic stress disorder among adult earthquake survivors in Peru. Disaster Medicine and Public Health Preparedness, 4, 39-46.

Cohen, R. (2008). Lecciones aprendidas durante desastres naturales. Revista Peruana de Medicina Experimental y Salud Pública, 25, 109-117.

Cova, F., \& Rincón, P. (2010). El terremoto y tsunami del 27-F y sus efectos en la salud mental. Terapia Psicológica, 28, 179-185.

Eksi, A., Braun, K.L., Ertem-Vehid, H., Peykerli, G., Saydam, R., Toparlak, D., \& Alyanak, B. (2007). Risk factors for the development of PTSD and depression among child and adolescent victims following a 7.4 magnitude earthquake. International Journal of Psychiatry in Clinical Practice, 11, 190-199.

Figueroa, R.A., González, M., \& Torres, R. (2010). Plan de reconstrucción psicológica post terremoto. Revista Médica de Chile, 138, 920-921.

Foa, E.B., Cashman, L., Jaycox, L., \& Perry, K. (1997). The validation of a self-report measure of posttraumatic stress disorder: The posttraumatic diagnostic scale. Psychological Assessment, 9, 445-451.

Foa, E.B., Johnson, K.M., Fenny, N.C., \& Treadwell, K.R. (2001). The child PTSD symptom scale: A preliminary examination of its psychometric properties. Journal of Clinical Child Psychology, 30, 376-384.

Goenjian, A.K., Pynoos, R.S., Steinberg, A.M., Najarian, L.M., Asarnow, J.R., Karayan, I., Ghurabi, M., \& Fairbanks, L.A. (1995). Psychiatric comorbidity in children after the 1988 earthquake in Armenia. Jour- 
nal of the American Academy of Child \& Adolescent Psychiatry, 34, 1174-1184.

Goenjian, A.K., Steinberg, A.M., Najarian, L.M., Fairbanks, L.A., Tashjian, M., \& Pynoos, R.S. (2000). Prospective study of posttraumatic stress, anxiety, and depressive reactions after earthquake and political violence. American Journal of Psychiatry, 157, 911-916.

Groome, D., \& Soureti, A. (2004). Post-traumatic stress disorder and anxiety symptoms in children exposed to the 1999 Greek earthquake. British Journal of Psychology, 95, 387-397.

Jiménez, A.E., \& Cubillos, R.A. (2010). Estrés percibido y satisfacción laboral después del terremoto ocurrido el 27 de febrero de 2010 en la zona centro-sur de Chile. Terapia Psicológica, 28, 187-192.

Kar, N., Mohapatra, P.K., Nayak, K.C., Pattanaik, P., Swain, S.P., \& Kar, H.C. (2007). Post-traumatic stress disorder in children and adolescents one year after a super-cyclone in orissa, India: Exploring cross-cultural validity and vulnerability factors. BMC Psychiatry, 7, 1-9.

Kovacs, M. (1981). Rating scales to assess depression in school-aged children. Acta Paedopsychiatria, 46, 305-315.

Kovacs, M. (1992). Children's depression inventory (CDI): Manual. North Tonawanda, NY: Multi-Health Systems.

Leen-Feldner, E.W., Feldner, M.T., Reardon, L.E., Babson, K.A., \& Dixon L. (2008). Anxiety sensitivity and posttraumatic stress among traumatic event-exposed youth. Behaviour Research and Therapy, 46, 548-556.

Leiva, M.C., \& Quintana G.R. (2010). Factores ambientales y psicosociales vinculados a síntomas de ataque de pánico después del terremoto y tsunami del 27 de febrero de 2010 en la zona central de Chile. Terapia Psicológica, 28, 161-167.

Li, X., Huang, X., Tan, H., Liu, A., Zhou, J., \& Yang, T. (2010). A study on the relationship between posttraumatic stress disorder in flood victim parents and children in Hunan, China. Australian and New Zealand Journal of Psychiatry, 44, 543-550.

Loubat, M., Fernández, A.M., \& Morales, M. (2010). La experiencia de Peralillo: Una intervención psicológica para el estado de emergencia. Terapia Psicológica, 28, 203-207.

Neria, Y., Besser, A., Kiper, D., \& Westphal, M. (2010). A longitudinal study of posttraumatic stress disorder, depression and generalized anxiety disorder in israeli civilians exposed to war trauma. Journal of Traumatic Stress, 23, 322-330.

Norris, F., \& Elrod, C. (2006). Psychosocial consequences of disaster. A review of past research. En F. Norris, S. Galea, M. Friedman, \& P. Watson (Eds.), Methods for Disaster Mental Health Research (p. 3-19). New York, USA: Guilford Press.

Norris, F.H., Friedman, M.J., \& Watson, P.J. (2002). 60,000 disaster victims speak: Part II. Summary and implications of the disaster mental health research. Psychiatry, 65, 240-260.

Norris, F.H., Friedman, M.J., Watson, P.J., Byrne, C.M., Diaz, E., \& Kaniasty, K. (2002) 60,000 disaster victims speak: Part I. An empirical review of the empirical literature, 1981-2001. Psychiatry, 65, 207-239.

Ortiz-Barrera, J., \& Manzo-García, C. (2010). Abordajes frente al terremoto y tsunami del 27 de febrero de 2010: Experiencia de la Sociedad Chilena de Psicología Clínica (SCPC). Terapia Psicológica, 28, 209-12.
Pérez-Benítez, C.I., Vicente, B., Zlotnick, C., Kohn, R., Johnson, J., Valdivia, S., \& Rioseco, P. (2009). Estudio epidemiológico de sucesos traumáticos, trastorno de estrés post-traumático y otros trastornos psiquiátricos en una muestra representativa de Chile. Salud Mental, 31,145-153.

Priebe, S., Grappasonni, I., Mari, M., Dewey, M., Petrelli, F., \& Costa, A. (2009) Posttraumatic stress disorder six months after an earthquake. Social Psychiatry and Psychiatric Epidemiology, 44, 393-397.

Reyes, H. (2010). Chile se pone de pie otra vez. Revista Médica de Chile, 138, 267-269.

Reynolds, C.R., \& Richmond, B.O. (1978). What I think and feel: A revised measure of children's manifest anxiety. Journal of Abnormal Child Psychology, 6, 271-280.

Reynolds, C.R., \& Richmond, B.O. (1985). The revised children's manifest anxiety scale: Manual. Los Angeles: Western Psychological Services.

Shoaf, K., \& Rottman, S. (2000). Public health impact of disasters. Australian Journal of Emergency Management, 15, 58-63.

Souza, M.D., \& Martínez, V. (2011). The intervention of TV in the chilean earthquake. Scientific Journal of Media Litreracy, 36, 69-76.

Stark, K.D., \& Laurent, J. (2001). Joint factor analysis of the Children's depression inventory and the revised children's manifest anxiety scale. Journal of Clinical Child Psychology, 30, 552-567.

Stefanis, C.N., \& Stefanis, N.C. (2002) Diagnosis of depressive disorders: A review. En Maj, M. \& Sartorius, N. (Eds.), Depressive Disorders (p. 1-87). New York: John Wiley \& Sons Ltd.

Sungur, M., \& Kaya, B. (2001). The onset and longitudinal course of a man-made post-traumatic morbidity: Survivors of the Sivas disaster. International Journal of Psychiatry in Clinical Practice, 5, 195-202.

Ticehurst, S., Webster, R.A., Carr, V.J., \& Lewin, T.J. (1996). The psychosocial impact of an earthquake on the elderly. International Journal of Geriatric Psychiatry, 11, 943-951.

Thienkrua, W., Lopes Cardozo, B., Somchai Chakkraband, M.L., Guadamuz, T.E., Pengjuntr, W., Tantipiwatanaskul, P., Sakornsatian, S., Ekassawin, S., Panyayong, B., Varangrat, A., Tappero, J.W., Schreiber, M., \& van Griensven, F. (2006). Symptoms of posttraumatic stress disorder and depression among children in tsunami-affected areas in southern thailand. Journal of the American Medical Association, 296, 549-559.

Tolin, D.F., \& Foa, E.B. (2006). Sex differences in trauma and posttraumatic stress disorder: A quantitative review of 25 years of research. Psychological Bulletin, 132, 959-992.

Vicente, B., Rioseco, P., Saldivia, S., \& Torres, S. (2002). Estudio chileno de prevalencia de patología psiquiátrica (DSM-III-R/CIDI) (ECPP). Revista Médica de Chile, 130, 527-536.

Vogel, E., \& Vera-Villarroel, P. (2010). Psychology and natural disasters: Earthquake and tsunami in Chile on February 27. Terapia Psicológica, $28,143-145$. 